Journal of Computer Science 5 (3): 221-225, 2009

ISSN 1549-3636

(C) 2009 Science Publications

\title{
Computer Aided Geometric Modeling of Twist Fiber
}

\author{
Keartisak Sriprateep and Adisak Pattiya \\ Faculty of Engineering, Mahasarakham University, Kantharawichai District, \\ Mahasarakham, Thailand 44150
}

\begin{abstract}
Problem statement: The objective of this study is to compare the methods for approximating the positions of twist fiber in yarn structures. Computer Aided Design (CAD) is applied using parametric equation, spline curve and NURBS curve. The ideal twist fiber and fiber migration are studied in 3 dimensions. Approach: The fiber positions in yarn structures were measured by trace fiber technique. The twist fiber in rayon yarn with 2.1, 3.0 and 6.0 twist factors was used as examples. The geometrical properties of twist fiber including curvature and torsion were applied in the analysis of all twist fiber examples. Each position was approximated along the length of yarn and then graphical representations of fiber are shown. Results: For ideal twist yarn, using parametric equation gives a smoother or better curve than when using the spline and NURBS curves. However, in the case of fiber migration, continuity of spline and NURBS curves are smoother than that of parametric equation. Conclusion/Recommendations: According to the comparison of the approximation method, parametric equation is most suitable for geometric modeling of ideal twist fiber, whereas the spline and NURBS curves were recommended for approximating the position of migrating fiber.
\end{abstract}

Key words: Computer aided design, twist fiber in yarn structure, curves approximation, geometrical properties

\section{INTRODUCTION}

The basic geometric properties of textiles such as fiber, yarn structures and fabric are related to the complexity in the arrangement of fiber in yarn structures and to the weaving of yarn structures when making fabric. Also, the mechanical properties of fiber, yarn structures and fabric are non-linear and anisotropic due to the combination of many tiny fibers for the production of yarn and due to the contacted surfaces between these fibers when being forced. Almost all of the theories studying and analyzing geometrical properties of single spun yarn structures depend on the geometry of ideal twisted yarn which was defined by Hearle et al. ${ }^{[3]}$ based on the coaxial-helix model. However, all of the single spun yarn structures would not be perfectly like the ideal twist; that is, there would be a small change of the position of fiber radius from the center of yarn (fiber migration) along its length. Therefore, the geometrical properties of the fiber migration would have a direct impact on the mechanical properties of yarn.

The study of fiber migration began when Morton and Riding $^{[5]}$ developed trace fiber technique. Subsequently, Hearle et al. ${ }^{[4]}$ and Treloar ${ }^{[10]}$ proposed the ideal fiber migration patterns to analyze the geometry of yarns with the assumption that the fiber migrates regularly and uniformly from the outside to the center of the yarn and then returns to the outside again, thus keeping constant the packing density. As a consequence, Tao ${ }^{[9]}$ proposed a mathematical model of the helix as sine wave pattern with variable radius and this model was used in the analysis of the geometrical and mechanical properties of a migrating fiber. Grishanov et al $^{[2]}$ developed a method for the analysis of positions of staple fiber yarn by using Markov process. Subsequently, a fiber migration pattern of fiber in yarn structures was introduced by Neckar et al. ${ }^{[6]}$ whose model was modified from that of Treloar ${ }^{[10]}$. Sriprateep and Bohez ${ }^{[8]}$ modified the model of $\mathrm{Tao}^{[9]}$ by extending parameters that can be applied to a wider variety of samples in the analysis of geometrical properties of fiber migration.

For the application of CAD in modeling fiber, yarn structures and fabric, there are several methods commonly used for approximating the position of twist fiber. Nevertheless, it is still not well-understood which method is most suitable for which type of fiber position. Therefore, it is the main purpose of the current paper to find out the advantages and disadvantages of each

Corresponding Author: Keartisak Sriprateep, Faculty of Engineering, Mahasarakham University, Kantharawichai District, Mahasarakham, Thailand 44150 
method and to recommend the method that is most suitable for each fiber migrating condition (ideal twist or migrating fiber). This is carried out by critical analysis based not only on the assessment of the twist fiber geometrical properties (curvature and torsion), but also on the implementation of CAD. The use of CAD is beneficial to this study because it allows the comparison of the fiber twist in 3D illustration. It also allows the geometric modeling of yarn structure as assemblies of many fibers ${ }^{[8]}$ rather than as a single line of yarn path, which would be more realistic in visual simulation and ultimately in the prediction of the mechanical properties of yarn and fabric.

\section{MATERIALS AND METHODS}

Computer-aided geometric modeling steps: Methods used for geometric modeling of twist fiber were carried out by the following steps:

Step 1: The fiber positions in yarn structures are measured by trace fiber technique. In this study, the coordinates of fiber with different twist factors $(2.1,3.0$ and 6.0) were used as example that the experimental results by Hearle and Gupta ${ }^{[4]}$. The distance between each fiber location was $\pi / 2$ continuously throughout the length of the yarn. The geometric properties of twist fiber can be represented in the form of curvature and torsion. A parametric model explaining the position of the continuous fiber is given by $r(\psi)=(\mathrm{R}(\psi) \cos (\psi \mathrm{Q})$, $\mathrm{R}(\psi) \sin (\psi \mathrm{Q}), \psi \mathrm{Qh} / 2 \pi)^{\mathrm{T}}$ where radius is $\mathrm{R}(\psi)$ and the number of twist turns is $\mathrm{Q}$ for $0 \leq \psi \leq 2 \pi$. The parametric speed is calculated from $|r(\psi)|=\sqrt{R^{2}(\psi)+Q^{2}} \equiv C$. Therefore, the curvature ( $\left.\kappa\right)$ and torsion $(\tau)$ are:

$\kappa=\sqrt{\frac{\mathrm{R}^{2}(\psi)}{\mathrm{C}^{4}}}$

$\tau=\frac{\mathrm{Q}}{\mathrm{C}^{2}}$

Step 2: The methods for approximating the position of fiber were applied using parametric equation, spline curve and NURBS curve in order to model the curve along the position of fiber obtained from the experiments.

Parametric equation: The parametric equation of the helix with radius $R$ and pitch length $h$ is given by Eq. 3 .

$$
\mathrm{P}(\mathrm{u})=\left[\mathrm{R} \cos 2 \pi \mathrm{u}, \mathrm{R} \sin 2 \pi \mathrm{u}, \frac{\mathrm{hu}}{2 \pi}\right]^{\mathrm{T}}, 0 \leq \mathrm{u} \leq 1
$$

The helix equations are as follows: $x=R \cos 2 \pi u, y=R \sin 2 \pi u, z=\frac{h u}{2 \pi}$. The helix equation can be written in many forms, but the value of various variables would be the same. In case that there is an approximation of fiber migration, only the radius and the pitch of each position approximated along the length of yarn would be different.

Spline curve: The Spline curve was applied to exhibit the geometric complexity of fiber in yarn structures ${ }^{[2]}$. The Spline curve would pass the approximated positions. Therefore, it is possible to approximate the value passing the positions of fiber curvature of each cross sectional area that is continuous through out the yarn structures. The cubic spline can be shown as follows:

$$
\begin{aligned}
& \mathrm{x}(\Delta)=\alpha_{0}+\alpha_{1}(\Delta \mathrm{t})+\alpha_{2}(\Delta \mathrm{t})^{2}+\alpha_{3}(\Delta \mathrm{t})^{3} \\
& \mathrm{y}(\Delta \mathrm{t})=\mathrm{b}_{0}+\mathrm{b}_{1}(\Delta \mathrm{t})+\mathrm{b}_{2}(\Delta \mathrm{t})^{2}+\mathrm{b}_{3}(\Delta \mathrm{t})^{3} \\
& \mathrm{z}(\Delta \mathrm{t})=\mathrm{c}_{0}+\mathrm{c}_{1}(\Delta \mathrm{t})+\mathrm{c}_{2}(\Delta \mathrm{t})^{2}+\mathrm{c}_{3}(\Delta \mathrm{t})^{3}
\end{aligned}
$$

where, $\Delta t \in\left[t_{i} ; t_{i}+1\right]$

The constant $\mathrm{aj}_{\mathrm{j}} \mathrm{b}_{\mathrm{j}, \mathrm{c}_{\mathrm{j}}}(\mathrm{j}=0,1,2,3)$ can be calculated from the coordination of each position.

NURBS curve: The fiber curve can be approximated by a curve called cubic NURBS curve, which is a parametric spline whose main components include the $2 \mathrm{D}$ or $3 \mathrm{D}$ control points. In addition, the weights of these points and a knot vector limit the effect of the control vertices onto a given segment of the curve. The NURBS basic function can be defined by the CoxdeBoor recursion formula. When considering the weights $\left(\mathrm{w}_{\mathrm{i}}\right)$, the NURBS can be defined based on the center of mass concept. Using the same concept, the point on NURBS curve for a parameter $t$ can therefore be written as:

$$
r(t)=\frac{\sum_{i=0}^{n_{p}-1} w i B_{i}(t) \cdot r i}{\sum_{l=0}^{n_{p}-1} w B_{1}(t)}=\sum_{i=0}^{n_{p}-1} B_{i}(t) \cdot r i, \quad 0 \leq t \leq 1
$$

The weight of control vertices can be less or greater than 1, if conic curves such as circle, ellipse, parabola and hyperbola are applied. In the case where 
the weight is 1 , the NURBS would be the B-spline. Giving data points $\mathrm{d}_{\mathrm{i}}$ where $\mathrm{i}=0,1, \ldots, \mathrm{m}$ and the coordinates include $\mathrm{x}, \mathrm{y}$ and $\mathrm{z}$ on each cross-sectional area of yarn, the approximation curve in the least squares sense is given by ${ }^{[7]}$ :

Minimize $\sum_{\mathrm{i}=0}^{\mathrm{m}}\left\|\mathrm{d}_{\mathrm{i}}-\mathrm{r}(\mathrm{t})\right\|^{2}$

Normally, the position of fiber is first given before letting the NURBS curve passing the given point as close as possible.

Step 3: To compare each method for approximating the positions of twist fiber as applied in step 2, each position is approximated along the length of yarn and then graphical representations of fiber are shown. The fiber curves were displayed in the form of solid model by sweeping along the curve. This can be carried out with the aid of computer software. The fiber diameter was $6 \mu \mathrm{m}$ and the software used for the solid model was SolidWorks. The positions of twist fiber in rayon yarn with 2.1, 3.0 and 6.0 twist factors are used as examples.

\section{RESULTS AND DISCUSSION}

Curvature and torsion of twist fiber: The curvature and torsion of fiber can be calculated based on Eq. 1 and 2. Figure 1 shows the curvature and torsion of fiber by using fiber coordinates from Table 1 . In the case of ideal twist fiber, the curvature is constant, but in the case of migrating fiber, the curvature is not constant. The geometric property, curvature, depends on the radius of fiber from the center of yarn and the parametric speed (C). Torsion would depend on the number of turns of twist (Q) and the constant value of parametric speed. Figure 1a shows that the curvature and the torsion of ideal twist fiber are constant. Figure 1b-d show the curvature and the torsion of migrating fiber with 2.1, 3.0 and 6.0 twist factors and the results would depend on the above-mentioned factors.
CAD modeling for ideal twist fiber: Ideal twist fiber is defined as the twist fiber whose radius from the center of the yarn is constant and the length of one turn of twist is constant along the yarn. Figure 2 shows computer aided solid model of ideal twist using parametric equation, spline curve and NURBS curve. Figure 2 shows that the parametric equation technique gives better results than the spline and NURBS curves. It can also be noticed that the techniques of spline and NURBS curves give similar results. In addition, increasing the number of approximation points would make the spline curve and the NURBS curve give closer results to the parametric equation.

CAD modeling for migrating fiber: In reality, the twist of fiber is not ideal; that is, the radius from the center of the yarn is not constant and the length of one turn of twist is not constant along the yarn. Table 1 shows various positions of fiber in three dimensions. The approximation of positions by parametric equation, spline curve and NURBS curve for fibers with 2.1, 3.0 and 6.0 twist factors is displayed by Fig. 3. Figure 3 also shows the solid model of oblique and front views of fiber obtained from each approximation technique. As can be seen from the pictures, the continuity of the curve generated by the parametric equation model is not smooth due to approximation of continuous points to the power of zero $\left(\mathrm{C}^{\circ}\right)$. Consequently, each continuous point of parametric equation would be linear. In the case of spline and NURBS curve, the equation is in cubic form. Therefore, each continuous point of the approximation would be non-linear. The continuity of each point would then be smoother than when using parametric equation. According to the graphic results, the discrepancy of the spline curve and NURBS curve method is rather small. The difference of these two methods is that the NURBS curve would have local control property; that is, when the positions of particular points change, the NURBS curve would be affected only around the migrating area. However,

\begin{tabular}{|c|c|c|c|c|c|c|c|c|c|c|c|c|}
\hline \multirow[b]{2}{*}{ Item } & \multicolumn{3}{|c|}{2.1 Twist factor } & \multicolumn{3}{|c|}{ 3.0 Twist factor } & \multicolumn{3}{|c|}{ 6.0 Twist factor } & \multicolumn{3}{|c|}{ Ideal twist } \\
\hline & $\mathrm{x}$ & $\mathrm{y}$ & $\mathrm{z}$ & $\mathrm{X}$ & $\mathrm{y}$ & $\mathrm{z}$ & $\mathrm{X}$ & $\mathrm{y}$ & $\mathrm{Z}$ & $\mathrm{X}$ & $\mathrm{y}$ & $\mathrm{Z}$ \\
\hline 1 & 10.5 & 0.0 & 0 & 6.3 & 0.0 & 0 & 20.1 & 0.0 & 0 & 10 & 0 & 0 \\
\hline 2 & 0.0 & 9.0 & 100 & 0.0 & 10.5 & 20 & 0.0 & 18.9 & 30 & 0 & 10 & 40 \\
\hline 3 & -5.4 & 0.0 & 180 & -9.6 & 0.0 & 90 & -13.8 & 0.0 & 60 & -10 & 0 & 80 \\
\hline 4 & 0.0 & -11.7 & 250 & 0.0 & -17.1 & 110 & 0.0 & -9.6 & 80 & 0 & -10 & 120 \\
\hline 5 & 12.0 & 0.0 & 320 & 15.0 & 0.0 & 170 & 7.5 & 0.0 & 110 & 10 & 0 & 160 \\
\hline 6 & 0.0 & 23.7 & 380 & 0.0 & 13.2 & 210 & 0.0 & 6.0 & 130 & 0 & 10 & 200 \\
\hline 7 & -21.0 & 0.0 & 440 & -9.6 & 0.0 & 240 & -7.5 & 0.0 & 160 & -10 & 0 & 240 \\
\hline 8 & 0.0 & -18.3 & 520 & 0.0 & -10.8 & 290 & 0.0 & -8.4 & 180 & 0 & -10 & 280 \\
\hline 9 & 14.4 & 0.0 & 570 & 9.0 & 0.0 & 340 & 12.3 & 0.0 & 190 & 10 & 0 & 320 \\
\hline
\end{tabular}




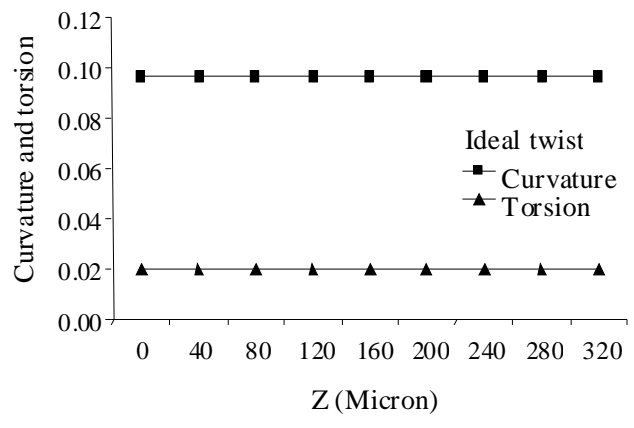

(a)

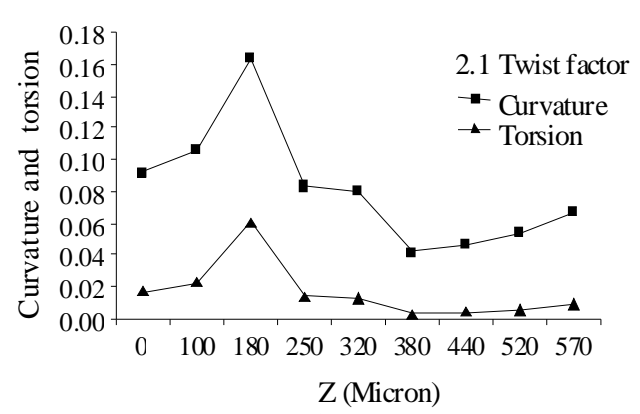

(b)

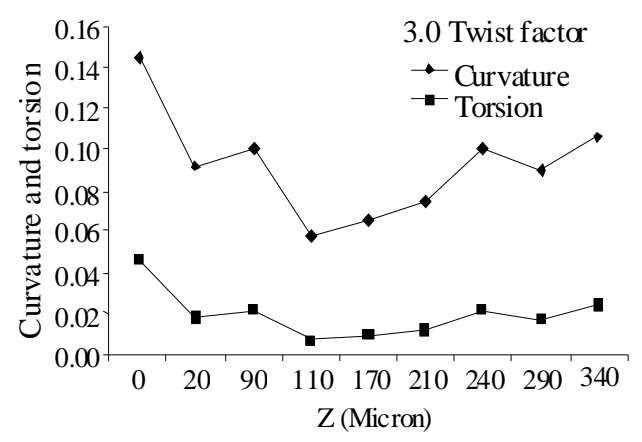

(c)

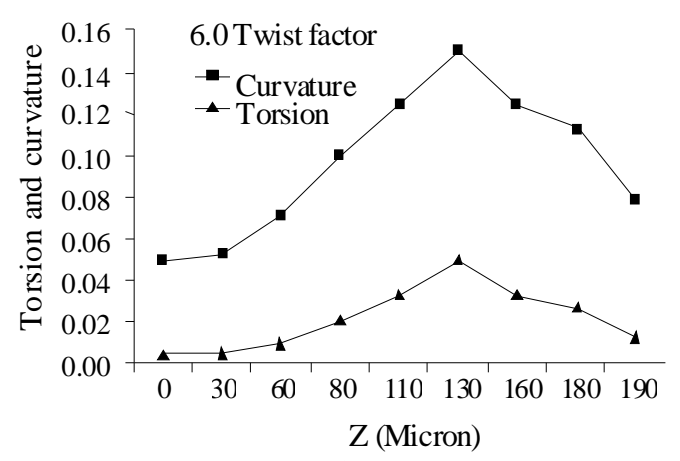

(d)

Fig. 1: Curvature and torsion of (a): Ideal twist fiber; (b-d): Migrating fiber of 2.1, 3.0 and 6.0 twist factors, respectively

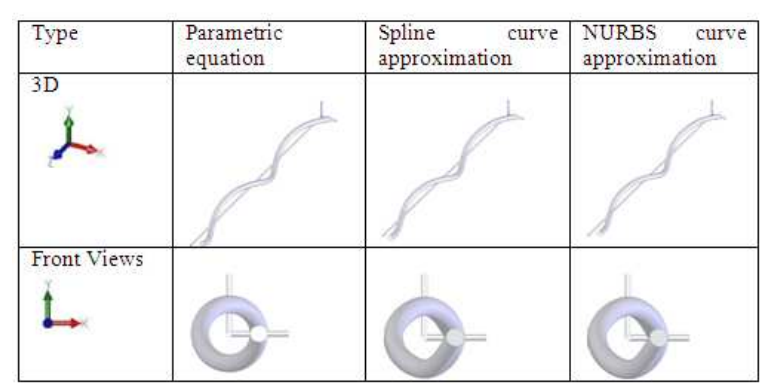

Fig. 2: Comparison of different methods in the case of ideal twist fiber

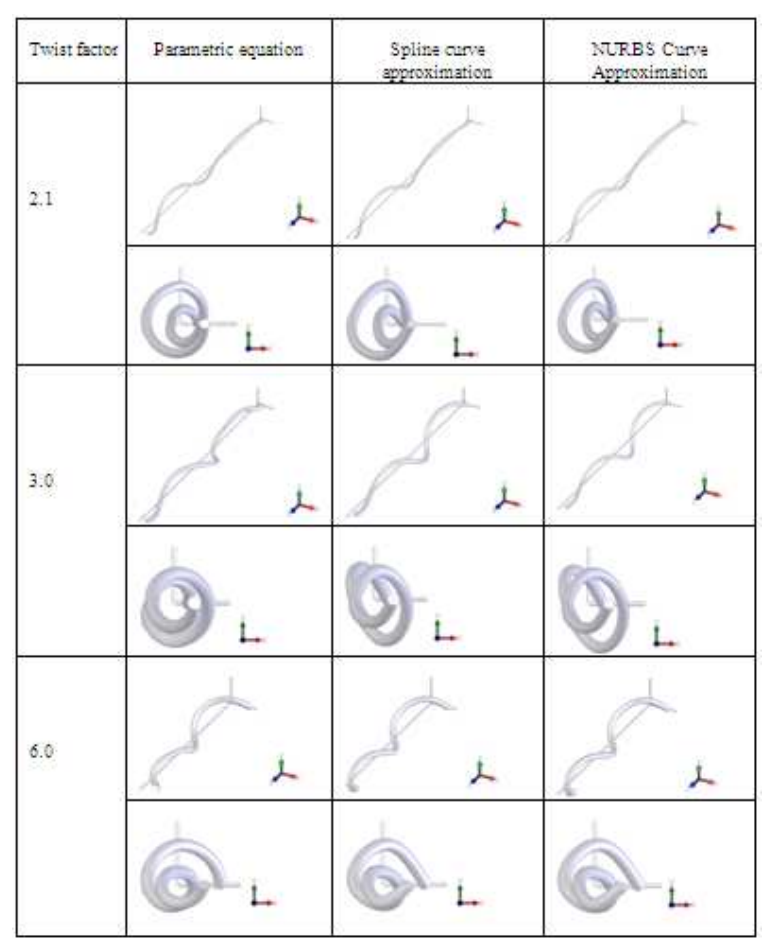

Fig. 3: Comparison of different methods in the case of migrating fiber

in the case of spline curve, when the positions of particular points change, all positions on such curve would be affected ${ }^{[1]}$. Accordingly, NURBS curve would be more flexible than spline curve.

\section{CONCLUSION}

According to the comparison of methods of approximation of positions using Computer Aided Design (CAD) in modeling geometry of twist of fiber in the yarn structures by using parametric equation, spline curve and NURBS curve. The positions of yarn 
structures from experimental results were used for yarn structures with $2.1,3.0$ and 6.0 twist factor. It was found that in case of ideal twist fiber, the approximation of positions by parametric equation rendered better smoothness and curvature of twist. However, in case of migrating fiber, spline curve and NURBS curve, the continuity of fiber would be smoother. The approximation of positions by parametric equation and spline curve would identify positions of fiber first then the curve passing specified position is determined directly. But, the method of NURBS curve would identify the position of fiber first then the NURBS curve would be determined to pass the specified position as close as possible. Therefore, NURBS curve is more flexible than spline curve for the application studied because NURBS is constructed piecewise so that changing a vertex affects the curve only in the vicinity of the changes control point, whereas in the case of spline curve, when the approximated position is changed, the result would affect all of the positions on the curve.

\section{ACKNOWLEDGEMENT}

Financial support from faculty of engineering, Mahasarakham University, Thailand, is gratefully acknowledged.

\section{REFERENCES}

1. Farin, G., 2002. Curves and Surfaces for CAGD: A Practical Guide. 5th Edn., Academic Press, San Francisco, ISBN: 978-1-55860-737-8.

2. Grishanov, S.A., R.J. Harwood and M.S. Bradshaw, 1999. A model fibre migration in staplefibre yarn. J. Textile Inst., 90: 298-321. http://cat.inist.fr/?aModele $=$ afficheN\&cpsidt $=10271077$
3. Hearle, J.W.S., P. Grosberg and S. Backer, 1969. Structural Mechanics of Fibers Yarns and Fabrics. 4th Edn., John Wiley and Sons, New York, ISBN: 10: 0471366692.

4. Hearle, J.W.S. and B.S. Gupta, 1965. Migration of fibers in yarns Part III: A study of migration in staple fiber rayon yarn. Textile Res. J., 56: 788-795. DOI: $10.1177 / 004051756503500902$

5. Morton, W.E. and K.C. Yen, 1952. The arrangement of fibers in fibro yarns. J. Textile Inst., 43: 60-66. 10.1080/19447025208659646

6. Neckar, B., M.K. Soni and D. Das, 2006. Modelling of radial fiber migration in yarns. Textile Res. J., 76: 486-491. DOI: 10.1177/0040517506061963

7. Piegl, L. and W. Tiller, 1997. The NURBS Book. 2nd Edn., Springer-Verlag Berlin Heidelberg, ISBN: 3540615458, pp: 646.

8. Sriprateep, K. and E.L.J. Bohez, 2009. A new computer geometric modeling approach of yarn structures for conventional ring spinning process. J. Textile Inst., 100: $14 . \quad$ DOI: 10.1080/00405000701757958

9. Tao, X., 1996. Mechanical propertied of a migrating fiber. Textile Res. J., 66: 754-762. DOI: 10.1177/004051759606601203

10. Treloar, L.R.G., 1965. A migration filament theory of yarn properties. J. Textile Inst., 56: 359-377. DOI: $10.1080 / 19447026508662296$ 